\section{El dengue y el dengue hemorrágico: prioridades de investigación}

\author{
María G. Guzmán, ${ }^{1}$ \\ Gissel García y Gustavo Kourí ${ }^{1}$
}

Palabras clave: dengue, revisión.

\footnotetext{
Instituto de Medicina Tropical Pedro Kourí, Ciudad de La Habana, Cuba. La correspondencia debe dirigirse a María G. Guzmán, Departamento de Virología, Centro Colaborador OPS/OMS para el Estudio del Dengue y su Vector, Instituto de Medicina Tropical Pedro Kourí, Autopista Novia del Mediodía, Km. 612/2, AP Marianao 13, Ciudad Habana, Cuba. Correo electrónico: lupe@ipk.sld.cu
}

El dengue es una enfermedad viral aguda causada por el virus del dengue y transmitida al hombre por el mosquito Aedes aegypti, su principal vector. De las enfermedades virales transmitidas por artrópodos, el dengue es la de mayor importancia actualmente y constituye una prioridad de salud pública en los países tropicales y subtropicales.

La incidencia y las epidemias de dengue han aumentado exponencialmente en los últimos 35 años a escala mundial. En el período de 1970 a 1998, el número de casos de dengue se cuadruplicó hasta alcanzar la cifra máxima de 1,3 millones, con más de 3600 muertes. Actualmente, el dengue es endémico en más de 100 países del sudeste asiático, el Pacífico occidental, América, África y el Medio Oriente.

Aunque no se ha determinado con exactitud la carga real de la enfermedad, se estima que de los 2500 millones de personas que viven en áreas en riesgo de transmisión, 50 millones se infectan anualmente y más de 500000 contraen su forma más grave, el dengue hemorrágico (DH). Durante las epidemias, las tasas de ataque pueden llegar a afectar a 80-90\% de las personas susceptibles (1) y la letalidad puede ser mayor de $5 \%(2,3)$.

El DH se describió por primera vez durante las epidemias de fiebre hemorrágica viral que ocurrieron en Manila, Filipinas, y en Bangkok, Tailandia, en la década de 1950. A partir de ese momento, el DH se extendió a otros países del sudeste asiático y del Pacífico occidental y posteriormente a las Américas. Si bien solo nueve países habían notificado casos de DH antes de 1970, en la actualidad se ha documentado su presencia en más de 30. Por ello se considera una enfermedad emergente, y como no se dispone de medios de control, constituye un problema creciente de salud que amenaza con propagarse a todo el mundo (1-5).

El impacto económico de las epidemias de dengue no se ha estudiado a fondo. Países como Cuba, Puerto Rico y Tailandia informan gastos de US\$ 6,8 a $\$ 103,0$ millones solo en atención médica, medidas para el control vectorial, horas de trabajo perdidas y pérdidas por la disminución del turismo (6-8). Se estima que en el período de 1984 a 1994 se perdieron 658 años de vida en Puerto Rico (9), pérdida similar a la informada en otras partes del mundo en conexión con esta enfermedad y con otros graves problemas de salud, como el paludismo y la tuberculosis.

Los factores determinantes de la aparición del $\mathrm{DH}$ y de la reemergencia del dengue son complejos 
y no se han estudiado completamente, aunque podrían estar relacionados con los profundos cambios demográficos y sociales que se produjeron durante la Segunda Guerra Mundial, los cuales favorecieron la propagación del virus y de su vector por varios países del sudeste asiático (10). Posteriormente, el crecimiento sin precedentes de la población, la urbanización masiva sin planificar, el insuficiente abastecimiento de agua potable, la disposición inadecuada de los residuos sólidos y de los depósitos no biodegradables, el aumento del número de viajeros y de las migraciones poblacionales, el deterioro de los sistemas de salud y de los programas de control, y la pobreza han contribuido a agravar la situación epidemiológica mundial. La aparición de cepas con mayor virulencia y capacidad de transmisión, así como la circulación simultánea de varios serotipos y genotipos en una misma región, también han influido en el agravamiento de las epidemias de dengue y de $\mathrm{DH}$ en diversas partes del mundo. Recientemente se demostró que los cambios climáticos de los últimos años contribuyen a la transmisión viral, por lo que se espera que en el futuro aumente el número de enfermos $(2,11-13)$.

En la Región de las Américas se ha observado claramente la emergencia y reemergencia del dengue. En este trabajo se analiza la situación epidemiológica actual de esta enfermedad en la Región y se examinan las principales iniciativas mundiales y regionales dirigidas a controlarla, así como las líneas de investigación que se deben aplicar en el futuro inmediato.

\section{EL DENGUE EN LAS AMÉRICAS}

En 1946, la Organización Panamericana de la Salud (OPS) inició un programa de control del mosquito $A$. aegypti con el objetivo de eliminar los focos de fiebre amarilla que aún quedaban en diversos países de la Región (14-16). A pesar del éxito logrado en su momento, con consecuencias positivas para el control de la fiebre amarilla y del dengue, la erradicación del vector fue difícil de mantener y a finales de la década de 1960 y principios de la de 1970 se constató la reinvasión del mosquito vector.

La segunda mitad del siglo XX marcó un cambio en la situación epidemiológica de las Américas. En 20 años, la Región pasó de tener una baja endemicidad —caracterizada por pocas epidemias, pocos países involucrados y pocos enfermos- a una situación de hiperendemia - con la circulación simultánea de varios serotipos, epidemias frecuentes, muchos países endémicos-, con numerosos casos y epidemias de DH (10). En el período de 1960 a 1980 se documentaron epidemias en el Caribe con el serotipo 3 (1962-1963), los serotipos 2 y 3 (1968-1969) y el serotipo 1 (1977-1978). Aunque la epidemia con el serotipo 1 comenzó en Jamaica, pronto se extendió a otras islas caribeñas, América Central y América del Sur, acompañada de la "desaparición" del serotipo 3, cuyos últimos aislamientos se detectaron en Puerto Rico y Colombia ese mismo año (1977) (17-20).

La década de 1980 representó un cambio en la historia del dengue. En 1981 se documentó en Cuba la primera epidemia de DH en la Región, causada por el serotipo 2 de un genotipo asiático. Durante esta epidemia se notificaron más de 300000 casos, de ellos más de 10000 graves o muy graves. Además, fallecieron 158 enfermos, entre ellos 101 niños (21). Cuando se analizan las cifras de casos de dengue y de DH en la Región, llama la atención la epidemia cubana por su extemporaneidad con relación al contexto epidemiológico histórico (figuras 1 y 2). Antes del año 1981, solo se habían informado 60 casos de DH en toda la Región y después de erradicada esa epidemia en octubre de ese mismo año, no se observaron prácticamente más casos de $\mathrm{DH}$ hasta que se desató una nueva epidemia en 1989 en Venezuela $(22,23)$.

A partir de ese momento ha aumentado el número de países que notifican casos de $\mathrm{DH}$ y la incidencia mantiene una tendencia ascendente (17). En 1981 se produjo también la introducción en la Región del serotipo 4, aislado primero en algunos países caribeños y luego en una gran parte de los países de América Latina y el Caribe.

Dos hechos importantes han ocurrido desde entonces que han agravado la situación epidemiológica en la Región:

a) la introducción del mosquito A. albopictus en 1985 procedente de Asia, que se extendió gradualmente por los países del área, aunque hasta el momento no se ha asociado con la transmisión de dengue (24)

b) la reintroducción del serotipo 3 del virus en América Central en 1994, que después se extendió a México, el Caribe y América del Sur y que produjo un incremento en las epidemias de dengue y en el número de casos de $\mathrm{DH}(25,26)$.

Se ha observado que el número de enfermos de dengue aumenta cíclicamente de forma epidémica cada 3-5 años, siempre con una tendencia ascendente. La mayor incidencia en la Región se observó en 2002, con más de 1 millón de casos, entre ellos 14000 de DH. Según datos de la OPS, en noviembre de 2005, 27 países habían notificado casos de dengue y de DH (figura 3) y en 14 de ellos circulaban dos o tres serotipos simultáneamente $(17,22)$. 
FIGURA 1. Casos de dengue en las Américas, 1980-2005a

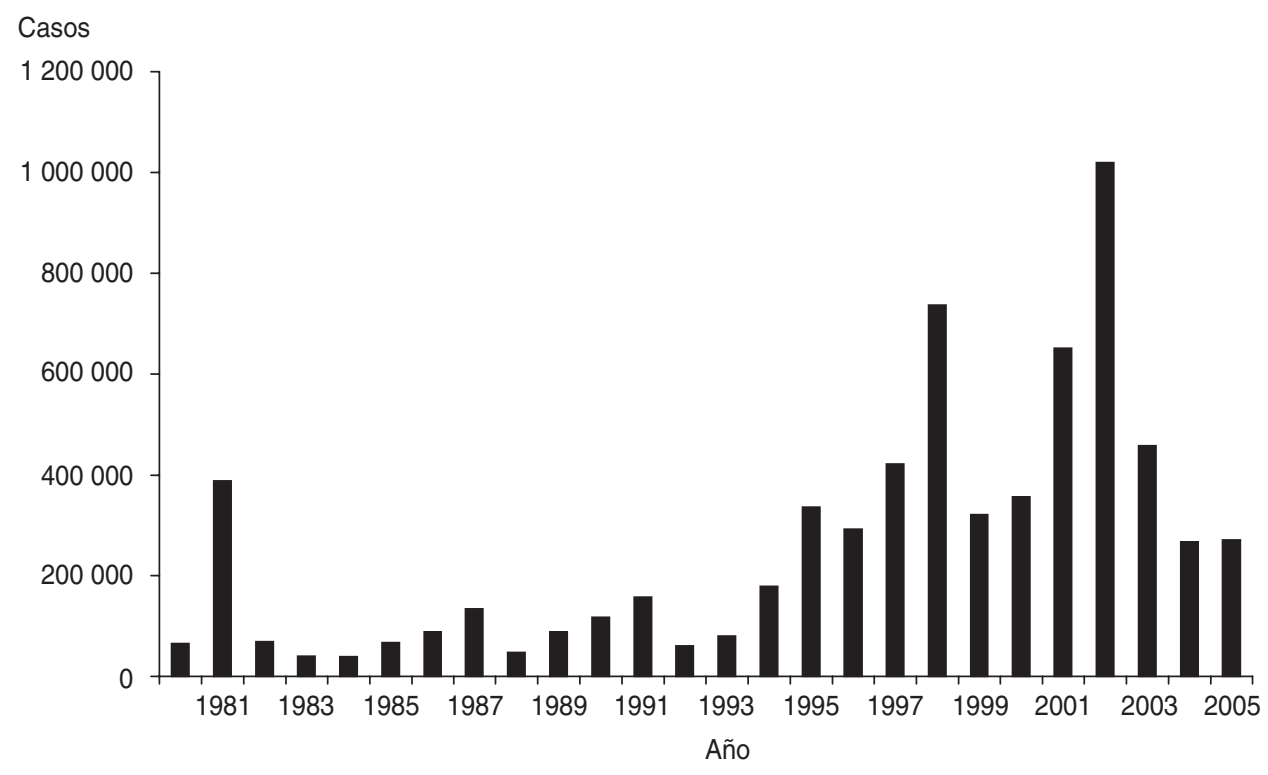

Fuente: Datos de la Organización Panamericana de la Salud (22, 23).

${ }^{a}$ Los datos de 2005 son de enero a noviembre.

FIGURA 2. Casos de dengue hemorrágico en las Américas, 1980-2005a

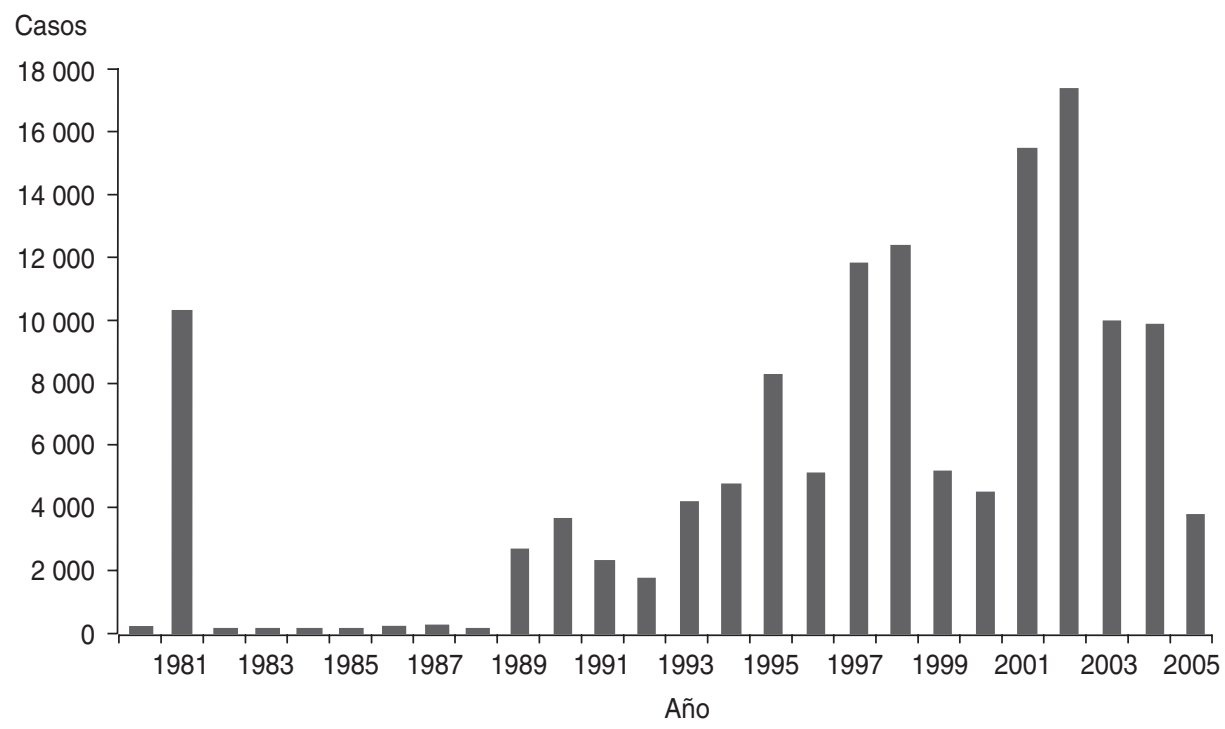

Fuente: Datos de la Organización Panamericana de la Salud (22, 23).

${ }^{a}$ Los datos de 2005 son de enero a noviembre.

\section{¿QUÉ SABEMOS?}

Teniendo en cuenta que la interacción entre el virus, el vector y el hospedero en un ecosistema de- terminado es en última instancia lo que determina la dinámica de la transmisión, es necesario realizar investigaciones que permitan lograr un mayor conocimiento de cada uno de estos factores con el ob- 


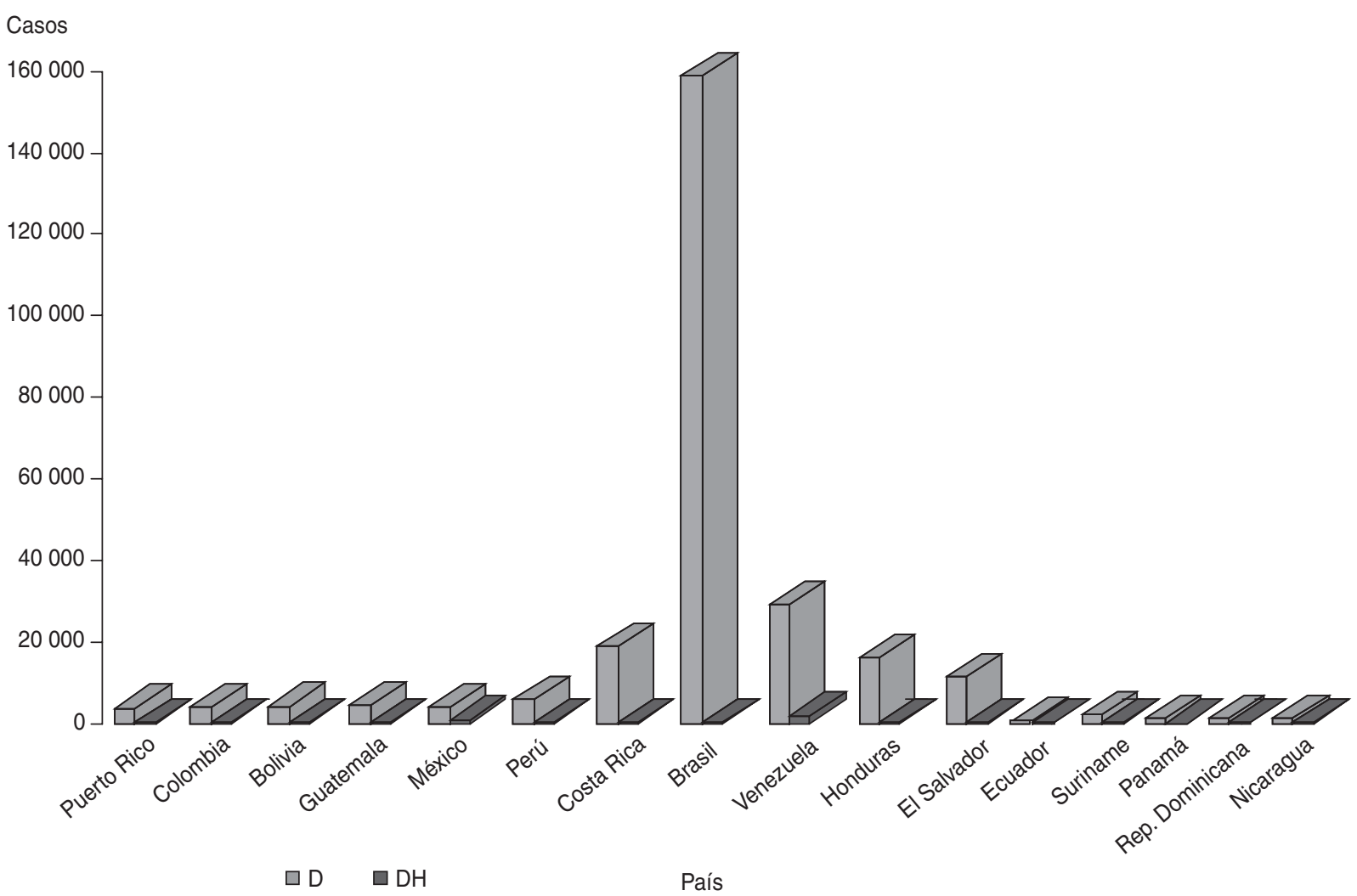

Fuente: Datos de la Organización Panamericana de la Salud (22). ${ }^{a}$ Los datos de 2005 son de enero a noviembre.

jetivo de reducir la morbilidad y la mortalidad por dengue y $\mathrm{DH}$, así como para aliviar la carga de estas enfermedades.

\section{El virus del dengue}

El virus del dengue está constituido por una cadena simple de alrededor de 11 kilobases $(\mathrm{kb})$ de ARN de polaridad positiva. Este complejo viral formado por cuatro serotipos (del Den 1 al Den 4) pertenece al género Flavivirus de la familia Flaviviridae. Su genoma codifica tres proteínas estructurales - la proteína $\mathrm{C}$, que compone la cápside que rodea y protege al ácido nucleico; la $\mathrm{M}$, que forma la membrana viral; y la E, que conforma la envoltura- y siete proteínas no estructurales (27). Se sabe que la glicoproteína $\mathrm{E}$ desempeña un papel fundamental durante la penetración del virus en la célula y en la respuesta inmunitaria (28). Por su parte, la proteína no estructural NS1 participa en la maduración viral y pudiera servir para el diagnóstico temprano.

Los cuatro serotipos del dengue son filogenéticamente diferentes y están más relacionados con los flavivirus transmitidos por mosquitos. Aunque se desconoce exactamente su origen, estudios recientes indican que surgieron hace aproximadamente 1000 años a partir de un virus de mono y que su transmisión al hombre ocurrió en el transcurso de los últimos 320 años. Algunas investigaciones sitúan su origen en el continente africano; otras lo colocan en Asia (29-32).

Recientemente se demostró la presencia de varias poblaciones virales (cuasiespecies) en un mismo hospedero (33) y se ha comprobado la recombinación entre cepas, posiblemente debido a la circulación simultánea de genotipos diferentes de un serotipo en un mismo hospedero $(34,35)$. Se desconoce el significado de estos hallazgos; sin embargo, la diversidad genética del virus del dengue puede ocasionar la aparición de cepas con mayor capacidad de replicación, de más fácil transmisión o más virulentas. Esta diversidad genética también pudiera asociarse con la aparición de cepas con determinantes antigénicos o tropismo alterados (36).

Los estudios dirigidos a conocer mejor los receptores virales y celulares son también de gran importancia. Según algunas investigaciones, el virus 
del dengue puede entrar en una célula a través de muchos receptores, entre ellos algunos específicos para este virus. Se cree que existen receptores celulares de primera, segunda y tercera líneas - muchos de ellos no están definidos completamenteque deben enlazarse a receptores virales localizados en determinados dominios de la proteína E relacionados con la penetración del virus en la célula y su fusión con la membrana celular (37).

\section{Clínica}

La definición de un caso de $\mathrm{DH}$ propuesta por la Organización Mundial de la Salud (OMS) ha sido de gran utilidad para la clasificación clínica de los pacientes. Sin embargo, cada vez se encuentran con mayor frecuencia enfermos que no cumplen totalmente con estos criterios $(38,39)$. Por otra parte, cada vez son más frecuentes las llamadas manifestaciones inusuales en enfermos de dengue, como la insuficiencia hepática, la miocardiopatía y los trastornos neurológicos (encefalitis y encefalopatía) $(40,41)$.

La aplicación de procedimientos clínicos estandarizados para la clasificación y el tratamiento de los enfermos de dengue debe ayudar a reducir la morbilidad y la mortalidad por esta enfermedad. Por esta razón, la evaluación de la clasificación actual en diferentes condiciones epidemiológicas mediante protocolos clínicos estandarizados constituye un campo de investigación prioritario (42). También se deben estudiar la utilidad de los signos de alarma clínicos y de laboratorio como marcadores de pronóstico del $\mathrm{DH}$; la aplicación y el significado de los estudios ultrasonográficos, y la comparación del cuadro clínico del $\mathrm{DH}$, tanto en niños y adultos como en pacientes de diferentes regiones (43). Estos estudios se deben complementar con la capacitación del personal de salud en los países con enfermedad endémica.

\section{Patogenia}

En 1987, Kourí y colaboradores publicaron su hipótesis integral que explica la aparición de las formas graves de la enfermedad (44). Con anterioridad se habían presentado dos hipótesis que se excluían mutuamente: la infección secuencial de serotipos diferentes y la de una relación entre la gravedad de la enfermedad y la virulencia de la cepa.

El factor de riesgo principal de sufrir $\mathrm{DH}$ es tener una segunda infección con un serotipo diferente del que causó la infección primaria. También se ha logrado consenso en que tener menos de 15 años de edad es un factor de riesgo de $\mathrm{DH}$ y se han propuesto otros, entre ellos el ser de la raza blanca y sufrir de enfermedades crónicas como el asma, la diabetes y la anemia de células falciformes (45-50).

En el curso de la infección primaria, el virus penetra en la célula diana mediante su unión a un receptor celular y se producen anticuerpos neutralizantes capaces de proteger por largo tiempo contra la reinfección con ese mismo serotipo y durante solo 2-3 meses contra los otros serotipos. En cambio, durante una infección secundaria con un serotipo heterólogo se forman complejos virusanticuerpos que penetran en las células del sistema fagocítico mononuclear (monocitos y macrófagos) gracias a la unión del fragmento constante de la inmunoglobulina -que forma parte del inmunocomplejo- a los receptores celulares del tipo gamma. Como consecuencia se infecta un mayor número de células y se favorece la diseminación viral. Este fenómeno se conoce como amplificación dependiente de anticuerpos $(51,52)$.

La activación de los linfocitos $\mathrm{T}$ y la producción de citocinas son también factores importantes en la patogenia del DH $(53,54)$. En la actualidad se sabe que después de una infección primaria se producen clones de células $\mathrm{T} \mathrm{CD}^{+}$y CD8 ${ }^{+}$efectoras y con memoria que son específicas para el serotipo infectante, aunque capaces de reconocer los restantes serotipos. En el curso de una segunda infección se activan los clones con memoria frente al nuevo serotipo y así se desencadena la respuesta inmunitaria. En los casos de DH se exacerba la activación y liberación de citocinas, lo que se relaciona con la mayor gravedad del cuadro clínico. También se ha demostrado que en los pacientes con $\mathrm{DH}$ se activa el sistema del complemento y en los casos graves se pueden detectar concentraciones elevadas de las proteínas C3 y C1q. Se plantea que los complejos virus-anticuerpos circulantes podrían ser los que activan la reacción en cascada del complemento $(55,56)$.

Es posible que el DH se acompañe de reacciones autoinmunitarias. Esto puede deberse a la presencia de anticuerpos contra la proteína viral E y contra la proteína viral NS1 (con reactividad cruzada con algunos factores de la coagulación y con las plaquetas, respectivamente). Estos anticuerpos pueden desempeñar un papel importante en el mecanismo patogénico durante la infección secundaria. (56-58).

Otro factor que se debe tomar en cuenta al analizar el complejo mecanismo patogénico del DH es la virulencia de la cepa infectante. En este sentido se ha reconocido la asociación de algunos genotipos, como los serotipos Den 2 y Den 3 de origen asiático ${ }^{2}$,

\footnotetext{
2 Los llamados genotipos americanos de estos dos serotipos no se han relacionado con el DH $(17,60)$.
} 
con casos y epidemias de DH. Recientemente se demostró que los enfermos con dengue presentan una menor carga viral que los enfermos con DH $(59,60)$. Nuevos estudios han demostrado la presencia de componentes determinantes de virulencia en la proteína E y en el extremo 3' del genoma viral (61-63).

El conocimiento de los mecanismos patogénicos de esta enfermedad ayudará a identificar los factores de riesgo de las formas más graves, a mejorar el tratamiento de los pacientes y a abordar más adecuadamente el desarrollo de nuevos fármacos antivirales y vacunas.

\section{Diagnóstico}

El conocimiento de la cinética de los diferentes marcadores del curso de la infección por dengue es de la mayor importancia para su diagnóstico. El virus puede detectarse en la sangre desde 2 ó 3 días antes del comienzo de la fiebre hasta 4 ó 5 días después de su desaparición. Los anticuerpos de la clase IgM contra el virus del dengue se pueden detectar en más de $95 \%$ de los casos a partir del quinto día de la enfermedad. En la infección primaria, los anticuerpos de la clase IgG se comienzan a detectar entre el $7 .^{\circ}$ y el $10{ }^{\circ}$ día de fiebre, mientras que en la infección secundaria se observa un incremento muy temprano de los anticuerpos IgG con títulos muy elevados a partir del segundo día de fiebre. En algunos casos de infección secundaria no se detectan anticuerpos $\operatorname{IgM}(23,64,65)$.

La detección de anticuerpos IgM contra el dengue es suficiente para considerar el caso como probable y se debe notificar a las autoridades de salud (23). Cuando se demuestra la seroconversión mediante la detección de anticuerpos IgG o cuando su título aumenta cuatro veces o más se considera confirmado el caso de dengue. Los antecedentes clínicos y epidemiológicos que acompañan al paciente son muy importantes para el diagnóstico final (23).

En la actualidad, el Programa Especial para la Investigación y Capacitación en Enfermedades Tropicales (TDR), la Iniciativa para la Vacuna del Dengue Pediátrico (PDVI) y la OMS y sus oficinas regionales auspician un proyecto para la evaluación de los sistemas de diagnóstico serológicos que se comercializan. Esta evaluación, que se realizará en laboratorios de las Américas y del sudeste asiático, permitirá definir los mejores sistemas diagnósticos en términos de sensibilidad, especificidad y facilidad de ejecución ${ }^{3}$.

Aunque estudios recientes demuestran que los títulos de anticuerpos de las clases IgA e IgE se

3 Se pueden encontrar más detalles en http://www.who.int/tdr/ elevan durante la infección aguda por dengue (64, $66,67)$, no se conoce el papel que desempeñan estos anticuerpos desde el punto de vista de la respuesta inmunitaria y la patogenia de la enfermedad. Se debe evaluar su valor diagnóstico y su posible utilidad para pronosticar la evolución a formas graves de la enfermedad.

La detección de antígenos de la envoltura viral constituye un método de diagnóstico temprano; sin embargo, su baja sensibilidad - principalmente en los pacientes con infección secundaria- no ha favorecido su aplicación en la práctica diagnóstica (68). La circulación de la proteína NS1 en la sangre desde el mismo comienzo de la enfermedad hace pensar que se puede utilizar para el diagnóstico temprano si se logra desarrollar un sistema que ofrezca valores adecuados de sensibilidad y especificidad (69).

El serotipo infectante puede determinarse mediante el aislamiento viral y su posterior identificación. El aislamiento se realiza mediante el cultivo de células de mosquitos, principalmente de una línea celular de $A$. albopictus, que luego se inocula con la muestra clínica del paciente. También se puede realizar la inoculación intratorácica de mosquitos, particularmente de Toxorhynchites amboinensis, que aunque es el método de mayor sensibilidad para aislar este virus, es menos empleado en la práctica diaria. Una vez aislado el virus, se pueden identificar los cuatro serotipos virales mediante pruebas de inmunofluorescencia indirecta con anticuerpos monoclonales específicos $(64,70,71)$.

Otros métodos de diagnóstico confirmatorio capaces de identificar el genotipo de la cepa infectante se basan en la amplificación y detección de secuencias específicas del genoma viral mediante la reacción en cadena de la polimerasa $(\mathrm{RCP})$ en sus diferentes variantes (ya sea anidada o en tiempo real). Para ello se utilizan principalmente segmentos del gen que codifica la proteína E ó de alguno de los genes que codifican algunas proteínas no estructurales $(64,72-74)$.

En la actualidad no se cuenta con sistemas de diagnóstico temprano suficientemente sencillos para utilizar en la atención primaria. Aunque mediante la RCP se puede llegar a un diagnóstico temprano, su elevado costo y su alta complejidad impiden que esté disponible en muchos hospitales e instituciones de salud de las zonas endémicas.

\section{Respuesta ante las emergencias}

Muchos países y las principales organizaciones internacionales han establecido estrategias para enfrentar la reemergencia del dengue y la emergencia del DH. Las investigaciones relacionadas con el virus, el hospedero, el vector y su interacción en un 
ecosistema determinado pueden ayudar a controlar esta enfermedad.

La estrategia general promovida por la OMS (75) desde 1995 para prevenir y controlar el dengue comprende cinco elementos básicos:

1) el control del vector con la participación de la comunidad y de todos los sectores de la sociedad

2) la vigilancia activa de la enfermedad, basada en la vigilancia clínica (con el apoyo de laboratorios capaces de detectar tempranamente las epidemias) y la vigilancia del nivel de infestación del vector (que permite monitorear y evaluar los programas de control)

3) la preparación para las emergencias, mediante planes de contingencia que contemplen la preparación del personal médico para situaciones urgentes, la hospitalización, la atención y el tratamiento de los enfermos y el control del vector

4) el entrenamiento y el fortalecimiento de la capacidad nacional para la vigilancia, el diagnóstico de laboratorio, el cuidado y el tratamiento de los casos, así como para el control del vector

5) la investigación sobre todos los temas relacionados con el control del vector.

En 1994, la OPS publicó las guías para la prevención y el control del dengue en las Américas, preparadas por un comité de expertos de la Región y del sudeste asiático. Estas guías contienen las pautas principales para establecer una adecuada vigilancia y lograr el control de las epidemias de dengue (23).

La voluntad política, la intersectorialidad, la participación de la comunidad —es decir, de todos los sectores de la sociedad y la población-y el reforzamiento y la aplicación de la legislación sanitaria son principios básicos para lograr el control de esta enfermedad. Según esas guías, cada país debe desarrollar su propia estrategia para la prevención, basada en un plan de control del vector con la participación de toda la comunidad. La vigilancia clínica y epidemiológica con apoyo del laboratorio y la vigilancia entomológica, que abarca el estudio de la resistencia del vector a insecticidas, deben ser los pilares principales de esa estrategia. El objetivo de la vigilancia es detectar tempranamente la introducción del virus en el país o el aumento de su circulación para poner en marcha rápidamente las medidas de control necesarias $(24,42)$.

A pesar del trabajo desarrollado en los países de la Región, no se ha logrado detener la tendencia creciente en el número de casos de dengue. En la actualidad se llevan a cabo varias iniciativas a escala regional y mundial. La Estrategia de Gestión Integrada (EGI), propuesta por la OPS, tiene como obje- tivo fortalecer los programas nacionales con vistas a reducir la morbilidad y mortalidad y la carga social y económica generada por las epidemias (76). Según la EGI, se debe preparar una ruta de acción basada en cinco áreas estratégicas: la comunicación social, la entomología, la epidemiología, el laboratorio y la atención del paciente. El objetivo de esa estrategia es modificar la conducta (individual y colectiva) con relación a los factores de riesgo de transmisión, tanto fuera como dentro del sector de la salud, y planificar y ejecutar los planes nacionales de forma intrasectorial, intersectorial e interprogramática ${ }^{4}$.

Varios países centro y sudamericanos se han incorporado a esta iniciativa que se basa en dos pilares fundamentales: los grupos de trabajo de dengue (conocidos como GT-dengue) y los planes de comunicación para el impacto conductual (COMBI). Los primeros, integrados por expertos de la Región en las áreas estratégicas mencionadas anteriormente, trabajan con los países en el desarrollo de EGI nacionales. El modelo de los COMBI se basa en una metodología que ayuda de manera estratégica y estructurada a planificar, poner en práctica y monitorear acciones de movilización y comunicación sociales para lograr y mantener resultados conductuales muy específicos relacionados con la prevención y el control de las enfermedades transmisibles, en particular del dengue (77).

En esta nueva línea de acción dirigida a controlar esta enfermedad se destaca la vigilancia ambiental con un enfoque basado en el ecosistema. Esta vigilancia y el consecuente reordenamiento del medio ambiente deben contribuir a eliminar las condiciones que favorecen la aparición de criaderos del vector. Cuando el medio está controlado y ordenado se reducen los niveles de infestación con el vector $\mathrm{y}$, consecuentemente, el riesgo de transmisión de dengue (42).

Diferentes organizaciones e iniciativas internacionales - particularmente el TDR, la Iniciativa para la Investigación en Vacunas, el PDVI, la OMS $\mathrm{y}$ sus oficinas regionales, el Centro Internacional para la Investigación y Desarrollo (CIID) de Canadá y el $6 .^{\circ}$ Programa Marco de Investigación de la Unión Europea- están desarrollando diversas actividades de alcance mundial con el objetivo de lograr las metas propuestas.

Entre los estudios actualmente en marcha se destacan los dirigidos a evaluar nuevos índices vectoriales - como el índice de pupas-y la utilidad de cortinas impregnadas para el control de A. aegypti, así como a identificar las barreras que impiden el

4 Se pueden encontrar más detalles en http://www.paho.org/Project. asp?SEL $=$ TP\&LNG $=E N G \& I D=41$ 
éxito de las intervenciones comunitarias ${ }^{5}$. Además se trabaja en la búsqueda de índices que midan el cambio conductual de la población y en la puesta en marcha de un sistema integrado de vigilancia con un enfoque ecosistémico de prevención y control.

DengueNet es otra iniciativa de alcance mundial que responde a la resolución de la OMS sobre la prevención y el control del dengue y del $\mathrm{DH}$, adoptada en mayo de 2002 durante la $55^{\mathrm{a}}$ Asamblea Mundial de la Salud (78). Este sistema basado en Internet tiene el objetivo de contribuir a la vigilancia mundial de esta enfermedad y permite obtener y analizar los datos epidemiológicos y virológicos de los países participantes y mostrar en fase temprana las tendencias epidemiológicas en el mundo. Además, muestra en tiempo real los indicadores más importantes, como la incidencia, las tasas de mortalidad por DH y la frecuencia y la distribución de los casos y serotipos circulantes ${ }^{6}$.

\section{Vacunas}

No hay duda acerca de la necesidad de contar con una vacuna contra el dengue que brinde inmunidad de larga duración contra los cuatro serotipos del virus. Sin embargo, diversos factores han impedido la elaboración de vacunas específicas contra el dengue, como la falta de un modelo animal que reproduzca la enfermedad, el aún incompleto conocimiento de su patogenia y el insuficiente financiamiento que han tenido las investigaciones sobre el dengue (79-81). La documentación de las formas graves de la enfermedad 20 años después de la infección primaria añade un nuevo reto al desarrollo de una vacuna eficaz $(82,83)$.

Se han adoptado diversas estrategias, como la búsqueda de vacunas convencionales con virus vivo atenuado o inactivado, vacunas recombinantes compuestas por proteínas obtenidas por ingeniería genética, vacunas quiméricas basadas en la preparación de clonos infecciosos y vacunas de $\operatorname{ADN}(79,84)$. La disponibilidad actual de varias vacunas candidatas constituye un gran avance en el desarrollo de vacunas contra el dengue y se cuenta con el apoyo de la PDVI y el TVR. Sin embargo, no se espera que se pueda contar con una vacuna contra el dengue en los próximos 5-10 años, por lo que en la actualidad, la erradicación del vector continúa siendo la única alternativa viable para controlar esta enfermedad ${ }^{6}$.

\footnotetext{
5 Se pueden encontrar más detalles en http://www.who.int/tdr/

6 Se pueden encontrar más detalles en http://www.who.int/vaccine_ research/en/
}

\section{RETOS}

En los últimos 10 años se ha avanzado notablemente en el conocimiento del dengue, en particular en el desarrollo de vacunas y en la caracterización de la respuesta inmunitaria y de las propiedades moleculares del virus. Por otro lado, los países han adquirido una mayor conciencia del peligro que representa esta enfermedad y del riesgo que corren nuestras poblaciones durante los próximos 20-30 años. No obstante, todavía hay muchos aspectos que se deben investigar.

Según el TDR, las investigaciones estratégicas que se deben llevar a cabo deben estar dirigidas a:

a) llevar a cabo investigaciones básicas para conocer mejor el virus del dengue, sus vectores y hospederos, así como la dinámica de la transmisión viral y los factores biológicos, sociales y económicos que pueden contribuir a mejorar la promoción de las intervenciones comunitarias

b) crear y perfeccionar nuevas técnicas y tecnologías, como vacunas y sistemas de diagnóstico, más eficaces

c) idear y perfeccionar nuevos métodos de intervención e indicadores para la vigilancia entomológica y el monitoreo y la evaluación de las intervenciones

d) crear nuevas estrategias terapéuticas y validar, perfeccionar y aplicar guías para el tratamiento del dengue y del DH $(85,86)$.

Los ministros de salud de la Región aprobaron por unanimidad una resolución sobre la prevención y el control del dengue y del DH durante el 43. ${ }^{\circ}$ Consejo Directivo de la OPS, celebrado en septiembre de 2001. Esta resolución ha servido de marco de referencia para una nueva generación de programas de prevención y control del dengue (87). Estos programas contemplan entre sus estrategias lograr un fuerte liderazgo gerencial, el apoyo político y financiero sostenible para dar continuidad a las intervenciones, el control selectivo del vector mediante una comunicación social eficaz, la participación comunitaria y el control ambiental dirigido a cambiar el comportamiento individual y colectivo con relación al medio ambiente y la vigilancia activa integrada basada en un sistema de información de salud eficiente y confiable. Además, como parte de esas estrategias, se debe lograr que la atención médica de los pacientes esté disponible dentro y fuera del sistema de salud, que se evalúe periódicamente la eficacia de las acciones aplicadas, que se mida el impacto económico de los componentes de los programas de prevención y control y, finalmente, que se establezcan mecanismos para la ca- 
pacitación continua de los recursos humanos en las ciencias sociales y biomédicas, tanto local como centralmente.

En resumen, las investigaciones básicas y aplicadas que pueden tener implicaciones positivas para el control del dengue deben cubrir los siguientes aspectos:

- En cuanto a la vigilancia, la epidemiología y el control

- definir la carga de la enfermedad y el impacto social y económico del dengue y del DH

- fortalecer la vigilancia con un enfoque ecosistémico

- desarrollar nuevos indicadores entomológicos y conductuales que reflejen adecuadamente el riesgo de transmisión y permitan conocer el impacto de las acciones de la comunidad

- desarrollar modelos matemáticos para predecir el riesgo de transmisión y de epidemias

- desarrollar y evaluar nuevas estrategias para el control del vector basadas en la participación de la comunidad, tanto en situaciones normales como de emergencia

- profundizar en el conocimiento de las propiedades biológicas y genéticas del vector y su interacción con el hospedero.

- En cuanto a los aspectos clínicos

- reevaluar la clasificación clínica del dengue y del DH y estandarizar las definiciones clínicas de los casos de manera clara y sencilla

- caracterizar los signos de alarma clínica y los marcadores de pronóstico

- evaluar la utilidad diagnóstica de las técnicas ultrasonográficas

- evaluar las manifestaciones clínicas inusuales en el curso del dengue

- elaborar guías para el tratamiento y el control clínico de los enfermos

- establecer planes de capacitación del personal sanitario.

- Con respecto a los mecanismos patogénicos

- estudiar el mecanismo de la inmunoamplificación viral y de las respuestas inmunitarias humoral y celular

- estudiar la respuesta innata al virus del dengue, así como los mecanismos de inhibición de esa respuesta, tanto por parte del virus como del hospedero

- definir el papel de los anticuerpos neutralizantes y de la respuesta de las células $\mathrm{T}$

- profundizar en el estudio del mecanismo de neutralización viral y el significado de los mutantes de escape de la neutralización
- estudiar la respuesta inmunitaria contra los diferentes serotipos y genotipos virales

- determinar las secuencias virales de infección y los intervalos entre las infecciones virales que puedan constituir factores de riesgo de $\mathrm{DH}$

- definir las células diana de la infección viral (células dendríticas, macrófagos, monocitos, linfocitos B, entre otras) y el papel de las células endoteliales en el dengue

- estudiar los mecanismos que conducen a la extravasación de líquidos y a la hemorragia en los pacientes con $\mathrm{DH}$

- determinar el papel de la autoinmunidad en el curso del dengue y del DH

- determinar los factores de riesgo, en particular el papel que puede desempeñar la susceptibilidad genética del hospedero.

- Con respecto a la interacción entre el virus y el hospedero

- profundizar en el conocimiento de la estructura del virus del dengue y de las funciones de las diferentes proteínas virales

- estudiar los mecanismos de entrada del virus a la célula, los receptores virales y celulares implicados y los mecanismo de fusión viral

- definir los factores virales que determinan la virulencia y profundizar en el conocimiento del tropismo y la interferencia virales

- evaluar las implicaciones de la diversidad genética del virus en el desarrollo de vacunas y de nuevos fármacos antivirales.

- En el diagnóstico

- desarrollar métodos de diagnóstico tempranos y asequibles para los países endémicos

- evaluar los sistemas comerciales disponibles

- desarrollar y evaluar protocolos sencillos para el diagnóstico molecular y la identificación de los serotipos infectantes

- desarrollar métodos de laboratorio que permitan identificar las respuestas primaria y secundaria.

- En relación con las vacunas

- evaluar y aplicar diferentes tecnologías de avanzada para el desarrollo de vacunas

- estudiar los mecanismos patogénicos e inmunitarios asociados con la acción eficaz de las vacunas

- identificar modelos animales apropiados para evaluar vacunas candidatas

- desarrollar métodos sencillos para el estudio de la inmunoamplificación y la neutralización virales 
o determinar la relación entre los diversos factores de neutralización y protección

- realizar estudios de campo sobre la infección por el virus del dengue y la evaluación de vacunas

- evaluar la seguridad, inmunogenia y eficacia de las vacunas candidatas que se desarrollen.

\section{CONCLUSIONES}

En la actualidad no se cuenta con otros medios para el control del dengue y de su forma más grave, el DH, que el control de su vector. La OMS y la OPS han llamado a todos los países a adoptar las medidas necesarias que contribuyan a disminuir la carga de la enfermedad y su impacto médico y socioeconómico. Se espera que una respuesta internacional coordinada y eficaz y el desarrollo de investigaciones epidemiológicas, clínicas y virológicas en las que se conjuguen los más avanzados métodos y técnicas permitan revertir la tendencia epidemiológica ascendente del dengue y coadyuven a su control.

\section{SYNOPSIS}

\section{Dengue and dengue hemorrhagic fever: research priorities}

Dengue is one of the most important infectious diseases in tropical and subtropical countries. At present, the only strategy available to reduce the incidence of dengue is vector control. The World Health Organization and the Pan American Health Organization have called on all nations to take the needed steps to help diminish the burden of this disease and its medical and socioeconomic impact. It is hoped that it will be possible to reverse the increase in dengue and help control its spread through a coordinated, effective international response, along with epidemiological, clinical, and virological research that brings together the most advanced methods and techniques. This piece summarizes the most up-to-date information on dengue, analyzes current epidemiologic trends in the Region of the Americas, discusses the main global and Western Hemisphere initiatives to control the disease, and presents the main areas of research that should be developed in the immediate future.

Key words: dengue, biomedical research.

\section{REFERENCIAS}

1. Calisher $\mathrm{CH}$. Persistent emergence of dengue. Emerg Infect Dis. 2005;11: 738-9.

2. Guzmán MG, Kourí G. Dengue: an update. Lancet Infect Dis. 2002;2:33-42.

3. Gubler DJ. The changing epidemiology of yellow fever and dengue, 1900 to 2003: full circle? Comp Immunol Microbiol Infect Dis. 2004;27:319-30.

4. Mairuhu ARA, Wagenaar J, Brandjes DPM, van Gorp ECM. Dengue: an arthropod-borne disease of global importance. Eur J Clin Microbiol Infect Dis. 2004;23:425-33.

5. Saker L, Lee K, Cannito B, Gilmore A, Campbell-Lendrum D. Globalization and infectious diseases: a review of the linkages. Geneva: WHO; 2004. (TDR/ STR/SEB/ST/04.2).

6. Guzmán MG, Kourí G, Bravo J, Triana C. Estimación de las afectaciones económicas causadas como consecuencia de la epidemia de dengue hemorrágico ocurrida en Cuba en 1981. Rev Cubana Med Trop. 1992;44:13-7.

7. Von Allmen SD, López-Correa RH, Woodall JP, Morens DM, Chiriboga J, Casta-Vélez A. Epidemic dengue fever in Puerto Rico, 1977: a cost analysis. Am J Trop Med Hyg. 1979;28:1040-4.

8. Valdés L, Vila J, Guzmán MG. Impacto económico de la epidemia de dengue 2 en Santiago de Cuba, 1997. Rev Cubana Med Trop. 2002;54:220-7.

9. Melzert MI, Rigau JG, Clark GG, Reiter P, Gubler DJ. Using disability adjusted life years to asses the economic impact of dengue in Puerto Rico: 1984-1994. Am J Trop Med Hyg. 1998;59:265-71.

10. Gubler DJ. Dengue and dengue hemorrhagic fever: its history and resurgence as a global public health problem. En: Gubler DJ, Kuno G, eds. Dengue and dengue hemorrhagic fever. New York: CAB International; 1997. Pp. 1-22.

11. Mackenzie JS, Gubler DJ, Petersen LT Emerging flaviviruses: the spread and resurgence of Japanese encephalitis, West Nile and dengue viruses. Nat Med. 2004;10:S98-109.

12. Gubler DJ, Reiter PM, Ebi KL, Yap W, Nasci R, Patz JA. Climate variability and change in the United States: potential impacts on vector-and rodent-borne diseases. Environ Health Perspect. 2001; 109:223-33.
13. Holmes EC, Burch SS. The causes and consequences of genetic variation in dengue virus. Trends Microbiol. 2000; 74:74-7.

14. Monath TP. Yellow fever and denguethe interactions of virus, vector and host in the re-emergence of epidemic disease. Semin Virol. 1994;5:133-45.

15. Schleissman DJ, Calheiros LB. A review of the status of yellow fever and Aedes aegypti eradication programs in the Americas. Mosq News. 1974;34:1-9.

16. Gubler DJ. Epidemic dengue/dengue hemorrhagic fever as a public health, social and economic problem in the 21st century. Trends Microbiol. 2002;2:100-3.

17. Guzmán MG, Kourí G. Dengue and dengue hemorrhagic fever in the Americas: lessons and challenges. J Clin Virol. 2003;27:1-13.

18. Pan American Health Organization. Dengue in the Caribbean, 1977. Washington, D.C.: PAHO; 1979. (Scientific Publication No. 375).

19. Boshell J, Groot H, Gacharna MG, Márquez G, González M, Gaitán MO, et al. Dengue en Colombia. Biomédica. 1986;6:101-6. 
20. Ventura AK, Hewitt CM. Recovery of dengue-2 and dengue- 3 viruses from man in Jamaica. Am J Trop Med Hyg. 1970;19:712-5.

21. Kourí GP, Guzmán MG, Bravo JR, Triana C. Dengue hemorrhagic fever/ dengue shock syndrome: lessons from the Cuban epidemic, 1981. Bull World Health Organ. 1989;67:375-80.

22. Pan American Health Organization. Number of reported cases of dengue and dengue hemorrhagic fever (DHF), Region of the Americas (by country and subregion). Washington, D.C.: PAHO; 2005. Hallado en http://www.paho. $\mathrm{org} / \mathrm{spanish} / \mathrm{ad} / \mathrm{dpc} / \mathrm{cd} /$ dengue.htm. Acceso el 28 de diciembre de 2005.

23. Pan American Health Organization. Dengue and dengue hemorrhagic fever in the Americas: guidelines for prevention and control. Washington, D.C.: PAHO, 1994. (Scientific Publication No. 548).

24. Centers for Disease Control and Prevention. Aedes albopictus infestation, United States, Brazil. MMWR. 1986;35: 493-5.

25. Guzmán MG, Vázquez S, Martínez E, Álvarez M, Rodríguez R, Kourí G, et al. Dengue in Nicaragua, 1994: reintroduction of serotype 3 in the Americas. Pan Am J Public Health. 1996;1:193-9.

26. Rocco IM, Kavakama BB, Santos CL. First isolation of dengue 3 in Brazil from an imported case. Rev Inst Med Trop. São Paulo. 2001;43:55-7.

27. Chambers TJ, Hahn CS, Galler R, Rice $\mathrm{CM}$. Flavivirus genome organization, expression and replication. Annu Rev Microbiol. 1990;44:649-88.

28. Rey FA. Dengue virus envelope glycoprotein structure: new insight into its interactions durng viral entry. Proc Natl Acad Sci USA. 2003;1000:6899-901.

29. Weaver SC, Barrett ADT. Transmission cycles, host range, evolution and emergence of arboviral disease. Nat Rev Microbiol. 2004;2:789-801.

30. Rudnick A. Ecology of dengue virus. Asian J Infect Dis. 1978;2:156-60.

31. Gaunt MW, Sall AA, de Lamballerie X, Falconar AKI, Dzhivanian TI, Gould EA. Phylogenetic relationship of flaviviruses correlate with their epidemiology, disease association and biogeography. J Gen Virol. 2001;82:1867-76.

32. Wang E, Ni H, Xu R, Barrett ADT, Warowich SJ, Gubler DJ, et al. Evolutionary relationship of endemic/epidemic and sylvatic dengue viruses. J Virol. 2000;74: 3227-34.

33. Wang WK, Lin SR, Lee CM, King CC, Chang SC. Dengue type 3 virus in plasma is a population of closely related genomes: quasispecies. J Virol. 2002;76: 4662-5.

34. Twiddy SS, Holmes EC. The extent of homologous recombination in members of the genus floavivirus. J Gen Virol. 2003;84:429-40.

35. Holmes EC, Twiddy SS. The origin, emergence and evolutionary genetics of dengue virus. Infection. Gen Evol. 2003; 3:19-28.
36. Chevillon C, Failloux AB. Questions on viral population biology to complete dengue puzzle. Trends Microbiol. 2003; 11:415-21.

37. Halstead SB, Heinz FX, Barrett ADT, Roehrig JT. Dengue virus: molecular basis of cell entry and pathogenesis. Vaccine. 2005;23:849-56.

38. Harris E, Videa E, Pérez E, Sandoval E, Téllez Y, Pérez MA, et al. Clinical, epidemiologic and virologic features of dengue in the 1998 epidemic in Nicaragua. Am J Trop Med Hyg. 2000;63: 5-11.

39. Phuong CXT, Nhan NT, Kneen R, Thuy PTT, Thien CV, Nga NTT, et al. Clinical diagnosis and assessment of severity of confirmed dengue infections in Vietnamese children: is the World Health Organization classification system helpful? Am J Trop Med Hyg. 2004; 70:172-9.

40. Solomon T, Dung NM, Vaughn DW, Kneen R, Thao LTT, Raengsakulrach B, et al. Neurological manifestations of dengue infection. Lancet. 2000;355: 1053-9.

41. Nguyen TL, Nguyen TH, Tieu NT. The impact of dengue haemorrhagic fever on liver function. Res Virol. 1997;148: 273-7.

42. Guzmán MG, Kourí G, Díaz M, Llop A, Vázquez S, González D, et al. Dengue, one of the great emerging health challenges of the 21st century. Expert Rev Vaccines. 2004;3:511-20.

43. Thulkar S, Sharma S, Srivastava DN, Sharma SK, Berry M, Pandey RM. Sonographic findings in grade III dengue hemorrhagic fever in adults. J Clin Ultrasound. 2000;28:34-7.

44 Kourí GP, Guzmán MG, Bravo JR. Why dengue haemorrhagic fever in Cuba? 2: An integral analysis. Trans R Soc Trop Med Hyg. 1987;81:821-3.

45. Halstead SB, Nimmannitya S, Cohen $\mathrm{SN}$. Observation related to pathogenesis of dengue hemorrhagic fever. IV. Relation of disease severity to antibody response and virus recovery. Yale J Biol Med. 1970;42:311-28.

46. Sangkawibha N, Rojanasuphot S, Ahandrik S, Viriyapongse S, Jatanasen S, Salitul $\mathrm{V}$, et al. Risk factors in dengue shock syndrome. A prospective epidemiologic study in Rayong, Thailand. Am J Epidemiol. 1984;120:653-69.

47. Thein S, Aung MM, Shwe TN, Aye M, Zaw A, Aye KM, et al. Risk factors in dengue shock syndrome. Am J Trop Med Hyg. 1997;56:566-72.

48. Guzmán MG, Kourí G, Valdés L, Bravo J, Álvarez M, Vázquez S, et al. Epidemiological studies on dengue in Santiago de Cuba, 1997. Am J Epidemiol. 2000; 152:793-9.

49. Bravo JR, Guzmán MG, Kourí GP. Why dengue haemorrhagic fever in Cuba? 1: Individual risk factors for dengue haemorrhagic fever/dengue shock syndrome (DHF/DSS). Trans R Soc Trop Med Hyg. 1987;81:816-20.
50. Guzmán MG. Sequential dengue infection. The Cuban experience. Dengue Digest. 2005;2(3):2.

51. Halstead SD. Immune enhancement of viral infection. Prog in Allergy. 1982;31: 202-9.

52. Morens DM, Halstead SD. Measurement of antibody dependent infection enhancement of four dengue virus serotypes by monoclonal and polyclonal antibodies. J Gen Virol. 1990;70:2909-14

53. Hober D, Poli L, Roblin B, Gestas P, Chungue E, Granic G, et al. Serum levels of tumour necrosis factor-alfa (TNF alfa), interleukin 6 (IL-6), and Interleukin 1 beta (IL-1beta) in dengue infected patients. Am J Trop Med Hyg. 1993;48:324-31.

54. Kurane I, Innis BL, Nimmannitya $S$, Nisalak A, Meager A, Janus J, et al. Activation of T-lymphocytes in dengue virus infection. High levels of soluble interleukin 2 receptor, soluble CD4, soluble CD8, interleukin 2, and interferon gamma in sera of children with dengue. J Clin Invest. 1991;88:1473-80.

55. Rothman AL, Ennis FA. Immunopathogenesis of dengue hemorrhagic fever. Virology. 1999;257:1-6.

56. Lei HY, Yeh TM, Liu HS, Lin YS, Chen $\mathrm{SH}$, Liu CC. Immunopathogenesis of dengue virus infection. J Biomed Sci. 2001;8:377-88.

57. Lin CF, Lei HY, Liu CC, Liu HS, Yeh TM, Wang ST, et al. Generation of IgM antiplatelet autoantibody in dengue patients. J Med Virol. 2001;63:143-9.

58. Markoff JL, Innis LB, Houghten R, Henchal SL. Development of cross-reactive antibodies to plasminogen during the immune response to dengue virus infection. J Infect Dis. 1991;164:294-301.

59. Rosen L. Disease exacerbation caused by sequential dengue infection: myth or reality. Rev Infect Dis. 1989;11:5840-2.

60. Rico-Hesse R, Harrison LM, Salas RA, Tovar D, Nisalak A, Ramos C, et al. Origins of dengue type 2 viruses associated with increased pathogenicity in the Americas. Virology. 1997;230:244-51.

61. Leitmeyer K, Vaughn D, Watts DM, Salas R, Villalobos de Chacon I, Ramos $\mathrm{C}$, et al. Dengue virus structural differences that correlate with pathogenesis. J Virol. 1999;73:4738-47.

62. Cologna R, Rico-Hesse R. American genotype structures decrease dengue virus output from human monocytes and dendritic cells. J Virol. 2003;77:3929-38.

63. Cologna R, Armstrong PM, Rico-Hesse $R$. Selection for virulent dengue viruses occurs in humans and mosquitoes. J Virol. 2005;79:853-9

64. Guzmán MG, Kourí G. Dengue diagnosis, advances and challenges. Int J Inf Dis. 2004;8:69-80.

65. Guzmán MG, Álvarez M, Vázquez S, Kourí $G$. Laboratory diagnosis of dengue infection: epidemiology and field studies. Dengue diagnostic: proceedings of an international workshop of UNICEF/ UNDP/World Bank/WHO Special Pro- 
gramme for research and Training in Tropical Diseases (TDR), 2004 October 4-6; Geneva. Geneva: WHO/TDR; 2005. (Documento TDR/IRM/DIAG/DEN/ 05.1, 2005)

66. Vázquez S, Pérez AB, Ruiz D, Rodríguez R, Pupo M, Calzada N, et al. Serological markers during dengue 3 primary and secondary infections. 2005;33:132-7.

67 Koraka P, Murgue B, Deparis X, Setiati TE, Suharti C, Gorp ECM, et al. Elevated levels of total and dengue virus-specific immunoglobulin $\mathrm{E}$ in patients with varying disease severity. J Med Virol. 2003; 70:91-8.

68. Kittigul L, Meethien N, Sujirarat D, Kittigul C, Vasanavat S. Comparison of dengue virus antigens in sera and peripheral blood mononuclear cells from dengue infected patients. Asian Pac J Allergy Immunol. 1997;15:187-91.

69. Alcon S, Talarmin A, Debruyne $M$, Falconar A, Deubel V, Flamand M. Enzyme-linked immunosorbent assay specific to dengue virus type 1 nonstructural protein NS1 reveals circulation of the antigen in the blood during the acute phase of disease in patients experiencing primary or secondary infections. J Clin Microbiol. 2002;40:376-81.

70. Vorndam V, Kuno G. Laboratory diagnosis of dengue virus infections. En: Gubler DJ, Kuno G, eds. Dengue and dengue hemorrhagic fever. New York: CAB International; 1997. Pp. 313-33.

71. Rodríguez R, Álvarez M, Guzmán MG, Morier L, Kourí G. Comparison of rapid centrifugation assay with conventional tissue culture method for isolation of dengue 2 virus in C6/36-HT cells. J Clin Microbiol. 2000;38:3508-10.

72. Deubel V. The contribution of molecular techniques to the diagnosis of dengue infection. En: Gubler DJ, Kuno G, eds. New York: CAB International; 1997. Pp. 335-66.
73. Rosario D, Álvarez M, Díaz J, Contreras R, Vázquez S, Rodríguez R, et al. Rapid detection and typing of dengue viruses from clinical samples using reverse transcriptase-polymerase chain reaction. Pan Am J Public Health. 1998;4:1-5.

74. Chutinimitkul S, Payungporn S, Theamboonlers A, Poovorawan Y. Dengue typing assay based on real-time PCR using SYBR Green I. J Virol Methods. 2005; 129:8-15.

75. World Health Organization. Key issues in dengue vector control toward the operationalization of a global strategy. Geneva: WHO; 1995. (Documento CTD/FIL DEN/IC/96.1).

76. San Martín JL, Prado M. Percepción del riesgo y estrategias de comunicación social sobre el dengue en las Américas. Rev Panam Salud Publica. 2004:15:135-9.

77. Parks W, Lloyd L. Planificación de la movilización y comunicación social para la prevención y el control del dengue. Guía paso a paso. Ginebra: Organización Mundial de la Salud, Centro Mediterráneo para la Reducción de Vulnerabilidad, Organización Panamericana de la Salud, Programa Especial de Investigación y Capacitación en Enfermedades Tropicales; 2004. (Documento WHO/ CDS/WMC/2004.2; TDR/STR/SEB/ DEN/ 04.1).

78. World Health Organization, Pan American Health Organization. DengueNet implementation in the Americas. Report of a WHO/PAHO/CDC Meeting; 2002 July 9-11; San Juan, Puerto Rico. Geneva: WHO; 2003. (Documento WHO/ CDS/CSR/GAR/2003.8); PAHO/HCP/ $\mathrm{HCT} / \mathrm{V} / 230 / 03)$

79. Guzmán MG, Mune M, Kourí G. Dengue vaccine: priorities and progress. Expert Rev Anti Infect Ther. 2004;2:1-17.

80. Hombach J, Barret AD, Cardosa, J, Deubel V, Guzmán MG, Kurane I, et al. Review on flavivirus vaccine develop- ment. Proceedings of a meeting jointly organized by the World Health Organization and the Thai Ministry of Public Health; 2004 April 26-27; Bangkok, Thailand. Vaccine. 2005;21:2689-95.

81. Halstead SB, Deen J. The future of dengue vaccines. Lancet. 2002;360: 1243-5.

82. Guzmán MG, Kourí G, Valdés L, Bravo J, Vázquez S, Halstead SB. Enhanced severity of secondary dengue-2 infections: death rates in 1981 and $1997 \mathrm{Cu}$ ban outbreaks. Pan Am J Public Health. 2002;11:223-7.

83. Peláez O, Guzmán MG, Kourí G, Pérez $\mathrm{R}$, San Martín JL, Vázquez $\mathrm{S}$, et al. Dengue 3 epidemic, Havana, 2001. Emerging Infect Dis. 2004;10: 719-22.

84. Pugachev KV, Guirakhoo F, Trent DW, Monath TP. Traditional and novel approaches to flavivirus vaccines. Int J Parasitol. 2003;33:567-82.

85. World Health Organization. Strengthening implementation of the global strategy for dengue fever and dengue haemorrhagic fever, prevention and control. Report of the Informal Consultation; 1999 October 18-20, Geneva, Switzerland. Geneva: WHO; 1999. (Documento WHO/CDS/DEN (IC)/2000.1)

86. World Health Organization. Meeting report of the Scientific Working Group on Dengue of the UNDP/World Bank/ WHO Special Programme for Research and Training in Tropical Diseases (TDR); 2000 April 3-5; Geneva, Switzerland. Geneva: WHO; 2000. (Documento TDR/DEN/SWG/00.1)

87. Organización Panamericana de la Salud. Nueva generación de programas de prevención y control del dengue en las Américas. Washington, D.C.: OPS; 2002. (Documento OPS/HCP/HCT/206/02). 